\title{
How the unicorn got its horn
}

An experiment studying bacterial populations over thousands of generations shows that a novel trait can evolve through rearrangement and amplification of a few pre-existing genes. SEE ARTICLE P.513

\section{HEATHER HENDRICKSON \& PAUL B. RAINEY}

$\mathrm{E}$ volutionary novelties such as flight, flowers and eyes are central to life's diversity, and yet explanations for their origins are anything but straightforward. The difficulty arises because the relevant events happened a long time ago under circumstances that are poorly understood. However, in this issue, Blount et al. ${ }^{1}$ (page 513) unravel molecular details underpinning a major innovation in a trait (phenotype) that evolved in bacterial populations after 30,000 generations. The capacity to store ancestral types in a state of suspended animation and then sequence their genomes has provided a remarkably complete picture of the main genetic events that gave rise to an instance of evolutionary novelty*.

The 'modern evolutionary synthesis', which provides the accepted account of evolution, unites Charles Darwin's gradualism - the view that evolution proceeds through gradual changes - with Gregor Mendel's concept of genes as discrete units. But some have argued that the resulting notion of incremental mutational change does not provide an adequate framework for explaining how phenotypic innovations arise $\mathrm{e}^{2,3}$. What steps precede a major evolutionary advance such as the eye? Far from being a simple philosophical point, the need for mechanistic understanding at this level is paramount, and Blount et al. (researchers in Richard Lenski's laboratory) provide direct insight into how innovation happens.

In 1988, Lenski initiated what is now the longest-running project in experimental evolution. He took a clone (a group of genetically identical cells derived from a single cell) of the fast-growing bacterium Escherichia coli and founded 12 independent populations, each resident in a flask containing a simple medium, with glucose as the sole source of carbon. Every day for almost 25 years, a sample of each population has been transferred to a fresh flask - which means that these populations have gone through 55,000 generations. In addition to daily transfer, the researchers have been periodically storing samples at $-80^{\circ} \mathrm{C}$, thereby ensuring

*This article and the paper under discussion ${ }^{1}$ were published online on 19 September 2012.

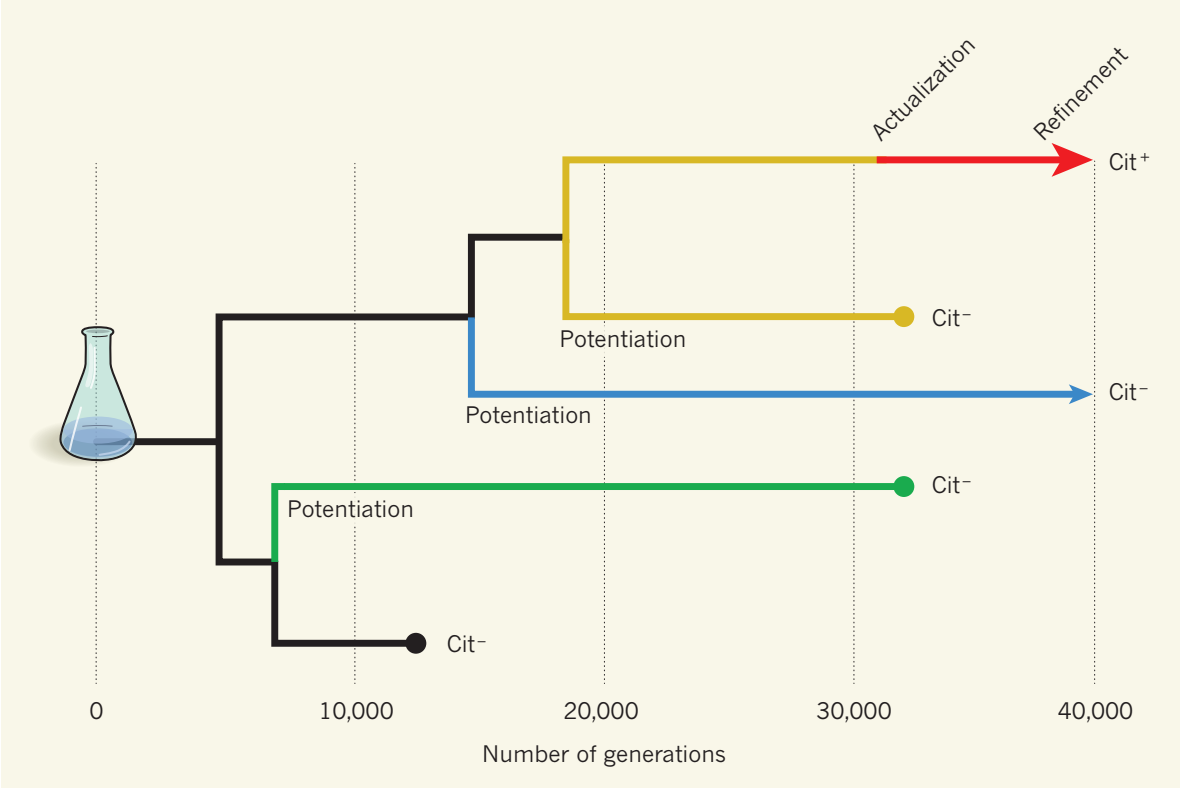

Figure 1 | The evolution of a new trait. In 1988, Richard Lenski took a single clone formed by genetically identical Escherichia coli bacteria and propagated it, with daily transfer, in 12 independent flasks. Whereas the original cells could use citrate as a nutrient only in the absence of oxygen ( $\mathrm{Cit}^{-}$cells), bacteria in one of the flasks eventually evolved the ability to use it with oxygen present (Cit ${ }^{+}$cells, indicated in red). After analysing the genomes of the microbes at different stages of the process, Blount et al. ${ }^{1}$ (researchers in Lenski's laboratory) found that the original clone had diverged into competing lineages - some of which went extinct and some of which prospered. The authors report that, for the new ability to appear, the bacterial populations went through three successive evolutionary steps. 'Potentiating' mutations (of unclear nature) were required for cells to acquire 'actualizing' mutations that consisted of a specific rearrangement of a few genes and that allowed some growth - although poor - on citrate in the presence of oxygen. Further 'refining' mutations, which involved duplications of the rearranged DNA sequence, were needed for robust growth under such conditions.

access to a detailed history of ancestral states. Moreover, unlike fossils, these frozen ancestors are readily brought back to life.

Although Lenski's group reported adaptations that improved the microbes' fitness from the earliest stages of the experiment, something remarkable happened after about 31,000 generations. One of the populations (Ara-3) evolved the capacity to use citrate as a nutrient ${ }^{4}$. Citrate, a chelating agent that facilitates iron uptake, is a component of the growth medium. Although E. coli can 'digest' citrate when oxygen is absent, it typically cannot do so under the aerobic conditions of this experiment. The fact that the microbe can use citrate under some circumstances raises an important point: new genes are not invented de novo. Quite the contrary - evolution builds on the raw material at its disposal. The bacterium has the building blocks necessary for the evolution of aerobic citrate utilization, but realization of this capacity requires a rewiring and refinement of regulatory connectivities.

To understand the nature of the genetic changes conferring the ability to use citrate in the presence of oxygen (a trait dubbed the $\mathrm{Cit}^{+}$phenotype), Blount et al. returned to the freezer and resuscitated ancestral $\mathrm{Cit}^{-}$populations from earlier time points in the Ara-3 lineage. When the researchers tried to 'replay' the evolution of the trait, they found that 
the $\mathrm{Cit}^{+}$phenotype appeared again only in populations derived from the most recent $\mathrm{Cit}^{-}$lineages. This result suggested that genetic changes had occurred in these lineages that made it possible for the $\mathrm{Cit}^{+}$phenotype to evolve. Although the precise nature of such 'potentiating' mutations is unclear, the important fact is that they took place (Fig. 1).

The step that made the cells weakly $\mathrm{Cit}^{+}$the 'actualization' step - was easier to identify. The authors examined a genomic region that carries the gene $\operatorname{cit} T$, which encodes a protein (citrate transporter) needed for citrate to enter the cell. In the original bacterial strain, cit $T$ is located downstream of cit $G$ (another gene required for citrate utilization) and $r n k$, a functionally unrelated gene that participates in energy metabolism. In each of the derived $\mathrm{Cit}^{+}$cells, Blount et al. found a distinctive genomic rearrangement that fused $r n k$ to $c i t G$. As a result, expression of $c i t G$ and cit $T$ came under the control of the promoter (regulatory sequence) of $r n k$, which allowed their expression in the presence of oxygen. What's more, the authors found that a single copy of the genetic rearrangement was insufficient to generate the $\mathrm{Cit}^{+}$phenotype, and that there was a tan-

"New genes are
not invented
de novo. Quite
the contrary:
evolution builds
on the raw
material at
its disposal."
dem array of between two and nine copies in $\mathrm{Cit}^{+}$cells. Therefore, gene duplications took a very low level of citrate transport and propelled it to life-sustaining levels. All cells that inherited the genetic fusion (the actualizing mutation), followed by amplification (a 'refining' mutation), were $\mathrm{Cit}^{+}$.

The discovery of the $\mathrm{Cit}^{+}$mutants in Lenski's experiment has been a mote in the eye for those suggesting that major phenotypic innovations cannot be explained by microevolutionary (gradual) processes. Indeed, for anti-evolutionists, lack of mechanistic detail has even allowed room for divine intervention. After several attempts to politely address the concerns of one critic, Lenski responded publicly in what has proven to be one of the most competent and direct defences of science versus dogma for some time. It includes the memorable phrase ${ }^{5}$, "In other words, it's not that we claim to have glimpsed 'a unicorn in the garden' - we have a whole population of them living in my lab!".

With knowledge of the key events that determine this unicorn's horn, one can next look for lessons of a general nature. The idea that genetic evolution might conform to a set of rules is attractive $e^{6}$. Capture and amplification of other genes' promoters have been shown to facilitate adaptation in a variety of situations $\mathrm{s}^{7,8}$, but the extent to which these kinds of events can fuel phenotypic innovation depends on prior history, chance and fortuitous factors such as genome architecture. Blount and colleagues' work shows that evolution, given sufficient time, will take advantage of any opportunity - but opportunity may depend on the most fickle of potentiating events. Unfortunately, predicting the nature of these crucial potentiating mutations may well lie beyond reach. Nevertheless, on a positive note, this work strengthens a central premise of the modern evolutionary synthesis: genetic change is gradual. Phenotypic change, however, can be dramatic.

Heather Hendrickson and Paul B. Rainey are at the New Zealand Institute for Advanced Study, Massey University, Auckland 0745,
New Zealand. P.B.R. is also at the Max Planck Institute for Evolutionary Biology, Plön,

Germany.

e-mail:p.b.rainey@massey.ac.nz

1. Blount, Z. D., Barrick, J. E., Davidson, C. J. \& Lenski, R. E. Nature 489, 513-518 (2012).

2. Pigliucci, M. Phil. Sci. 75, 887-898 (2008).

3. Gould, S. J. The Structure of Evolutionary Theory (Belknap, 2002)

4. Blount, Z. D., Borland, C. Z. \& Lenski, R. E. Proc. Natl Acad. Sci. USA 105, 7899-7906 (2008).

5. http://rationalwiki.org/wiki/Lenski_affair

6. Stern, D. L. \& Orgogozo, V. Science 323, 746-751 (2009).

7. Whoriskey, S. K., Nghiem, V. H., Leong, P. M., Masson, J. M. \& Miller, J. H. Genes Dev. 1, 227-237 (1987).

8. Andersson, D. I. Science 282, 1133-1135 (1998).

\title{
Spinning towards scalable circuits
}

\begin{abstract}
Silicon devices form the backbone of modern computers. It turns out that they might also be a natural hardware platform for a new era of computing technology that uses the principles of quantum physics. SEE LETTER P.541
\end{abstract}

\section{LEE C. BASSETT \& DAVID D. AWSCHALOM}

$\mathrm{M}$ ore than 50 years ago, the invention of integrated circuits based on silicon transistors (tiny electronic switches) revolutionized electrical and computer engineering. It allowed room-sized computers made of vacuum tubes to be replaced with individual semiconductor chips, which have continued to become exponentially smaller and more powerful. Now, a new kind of computing that relies on quantum rather than classical physics has the potential to crack problems inaccessible to even the most powerful classical computers of the foreseeable future. However, we are still searching for the best physical architecture for such quantum machines - even a suitable 'quantum vacuum tube', let alone the transistor equivalent, remains elusive.

Among many candidates for the quantum bits (qubits) of information that form the basis of quantum computing, the spins of single electrons or isolated nuclei in host semiconducting materials offer great potential for incorporation into large-scale devices. Silicon is a particularly attractive host material, in no small part because of its use in the well-developed microchip industry. On page 541 of this issue, Pla et al. ${ }^{1}$ report the first fully functional electron-spin qubit based on a single phosphorus atom placed in a silicon device ${ }^{\star}$.

*This article and the paper under discussion ${ }^{1}$ were published online on 19 September 2012.
In addition to the two allowed states, 0 or 1 , of classical bits (for example, the on/off states of transistors in a processor, or the magnetization direction of magnetic domains on a hard disk), a qubit, being quantum mechanical, can also exist in an arbitrary superposition of 0 and 1. Quantum mechanics also allows for non-trivial correlations between the states of multiple qubits, whereby measurement of one qubit alters the states of the others. A reasonably sized quantum machine could leverage the vastly increased number of states offered by superpositions, along with non-classical qubit correlations, to solve certain types of problem much more efficiently than any classical computer.

Many important problems fall into this category, including Peter Shor's famous algorithm ${ }^{2}$ to find a number's prime factors, and the simulation of complex quantum systems. The large-scale implementation of Shor's algorithm would fundamentally alter data encryption, and quantum simulators have the potential to revolutionize physics, chemistry and materials science, and therefore to drive advances in engineering, biology and medicine.

The prototypical qubit is the spin of an elementary particle such as an electron that can point either 'down' (0) or 'up' (1). Even ignoring quantum effects, these two spin states can be used as the logical states for classical information processing - this is the essence of 'spintronics'. But quantum computation also requires full control of superposition 